\title{
Blind decomposition of low-dimensional multi-spectral image by sparse component analysis
}

\author{
Ivica Kopriva ${ }^{1}$, and Andrzej Cichocki ${ }^{2,3}$ \\ ${ }^{1}$ Division of Laser and Atomic Research and Development, Ruđer Bošković Institute \\ Bijenička cesta 54, HR-10000, Zagreb, Croatia \\ phone: +385-1-4571-286, fax:+385-1-4680-104 \\ e-mail: ikopriva@irb.hr \\ ${ }^{2}$ Laboratory for Advanced Brain Signal Processing \\ Brain Science Institute, RIKEN 2-1Hirosawa, Wako-shi, Saitama, 351-0198, Japan \\ ${ }^{3}$ Warsaw University of Technology and Systems Research Institute, PAN, Poland. \\ e-mail: cia@brain.riken.jp
}

\begin{abstract}
Multilayer hierarchical alternating least square nonnegative matrix factorization approach has been applied to blind decomposition of low-dimensional multi-spectral image. Performance of the algorithm is invariant with respect to statistical (in)dependence between materials present in the image that is an assumption upon which many existing blind source separation methods depend. The proposed method performs blind decomposition exploiting spectral diversity and spatial sparsity between the materials present in the image. Unlike many existing blind source separation methods the method is capable to estimate the unknown number of materials present in the image. This number can be less than, equal to or greater than the number of spectral
\end{abstract}


accepted for Journal of Chemometrics

bands. Performance of the method is evaluated on underdetermined blind source separation problems associated with blind decompositions of experimental red-greenblue images composed of four materials. The proposed algorithm showed best performance in comparison with methods based on $\ell_{1}$-norm minimization: linear programming and interior-point methods. In addition to tumor demarcation problem demonstrated in the paper, other areas that can also benefit from proposed method are cell and chemical imaging.

Key words: Multi-spectral imaging, Chemical imaging, Cell imaging, Sparse component analysis, Nonnegative matrix factorization.

\section{INTRODUCTION}

Due to the variety of potential civilian and military applications, blind decomposition of multi-spectral and hyper-spectral images has drawn considerable attention in recent years [1-8]. Owing to the fact that more than one material is expected to be present in each pixel footprint, blind multi-spectral image decomposition is a blind source separation (BSS) problem. A standard tool for the solution of the BSS problems is independent component analysis (ICA) [9-13], which is based on the assumption that materials are mutually statistically independent. However, as shown in [14], this assumption is not fulfilled for the hyper-spectral and multi-spectral data due to the fact that sum of the materials present in the pixel footprint must be constant [18]. Thus, materials must necessarily be statistically related. ICA also requires the unknown number of materials present in the image to be less than or equal to the number of spectral bands. This assumption is easily satisfied in the case of hyper-spectral imaging but is problematic when low-dimensional multi-spectral images are considered. 
accepted for Journal of Chemometrics

Therefore, we propose an algorithm for blind decomposition of the low-dimensional multi-spectral images. Its performance does not depend on statistical relations among materials. The algorithm exploits spectral diversity of the materials present in the image as well as sparsity between their spatial distributions. The sparsity assumption implies that only small number of materials occupies the pixel footprint. This constraint is necessary to narrow down infinite number of factorizations that satisfy linear mixture model of the multi-spectral image (it is defined in section 2). Methods that solve BSS problems based on sparseness assumption are known as sparse component analysis (SCA) [15-17]. They solve related BSS problem in two phases: using data clustering algorithm to estimate mixing matrix] and using $\ell_{1}$-norm minimization to estimate spatial distributions of the materials present in the image. Geometrical methods proposed in $[18,19]$ for non-negative BSS problems, cannot solve underdetermined BSS problems. This is in principle also true for nonnegative matrix and tensor factorization (NMF/NTF) methods [20-25], which exhibited poor performance in solving underdetermined BSS problems. This limitation has been alleviated recently through introduction of the local or hierarchical NMF algorithm [23, 25]. It sequentially minimizes local cost functions in the source recovery process. Because it employs alternating least squares minimization to estimate mixing matrix and matrix of the materials it is coined as HALS NMF algorithm. When employed in multilayer mode [26], the HALS NMF algorithm has demonstrated good performance in solving underdetermined BSS problems. Thus, the multilayer HALS NMF algorithms and its application in blind multi-spectral image decomposition represent the main contributions of this paper. More details about this algorithm are presented in section 3. It capability to solve underdetermined BSS problem is demonstrated in section 4 on challenging problems of blind decomposition of experimental red-green-blue (RGB) 
accepted for Journal of Chemometrics

images. Very few algorithms exist for the solving blind multi-spectral image decomposition problem in underdetermined BSS scenario.

The rest of the paper is organized as follows. Section 2 introduces the static linear mixture model of the multi-spectral image. Section 3 presents HALS NMF (SCA)-based approach to blind multi-spectral image decomposition. Section 4 presents results of comparative performance analysis between multilayer HALS NMF algorithm and linear programming and interior point based $\ell_{1}$-norm minimization methods. Conclusions are given in section 5.

\section{STATIC LINEAR MIXTURE MODEL OF THE MULTI-SPECTRAL IMAGE}

Multi-spectral image is represented in a form of the static linear mixture model, [1-6]:

$$
\mathbf{X}=\mathbf{A S}
$$

where $\mathbf{X} \in \mathrm{R}_{0^{+}}^{N \times T}$ represents multi-spectral image consisting of $N$ spectral bands and $T=P \times Q$ pixels, $\mathbf{A} \in \mathrm{R}_{0^{+}}^{N \times M}$ represents mixing matrix or matrix of spectral responses and $\mathbf{S} \in \mathrm{R}_{0^{+}}^{M \times T}$ represents matrix of the $M$ materials present in the image scene. Each row of $\mathbf{X}$ and $\mathbf{S}$ is a 1D image representation obtained from corresponding spectral image by some $2 \mathrm{D} \rightarrow 1 \mathrm{D}$ mapping called vectorization. Because we are concerned with an unsupervised image decomposition problem $\mathbf{X}, \mathbf{A}$ and $\mathbf{S}$ are assumed to be nonnegative.

Unsupervised decomposition of the linear mixture model (1) is a challenging BSS problem because both mixing matrix $\mathbf{A}$ and materials matrix $\mathbf{S}$ must be estimated having at disposal multi-spectral image matrix $\mathbf{X}$ only. Many BSS problems are efficiently solved by ICA [9-13] assuming materials $\left\{\mathbf{s}_{m}\right\}_{m=1}^{M}$ to be statistically independent and non-Gaussian as well as that the number of materials $M$ is less than or 
accepted for Journal of Chemometrics

equal to the number of spectral bands $N$. As discussed in introduction, these assumptions are not fulfilled in low-dimensional multi-spectral imaging scenario. In such scenario estimating the number of materials $M$ is a challenging problem as well. Many approaches based on ranking of the singular values of the sample data covariance matrix $\mathbf{R}_{\mathbf{X}} \approx \mathbf{X} \mathbf{X}^{\mathrm{T}}$ [27-29] can not be used, because number of singular values $N$ is less than the number of materials $M$. In proposed HALS NMF algorithm we treat $M$ as unknown variable that is estimated together with the mixing matrix $\mathbf{A}$ by to be described data clustering algorithm [17].

\section{SCA-BASED BLIND MULTI-SPECTRAL IMAGE DECOMPOSITION}

When linear system of equations (1) is underdetermined the nullspace of $\mathbf{A}$ is nontrivial. Thus, the inverse problem has many solutions and additional constraints such as sparseness between the components of the column vectors $\{\mathbf{s}(t)\}_{t=1}^{T}$ are necessary to narrow down number of solutions. SCA concept is used to find a good approximation of the true solution to an underdetermined system of linear equations subject to sparsity constraints. A sparse signal is a signal with just few percent of the samples that are nonzero. Signal that has at least $k \leq M$ nonzero components is called $k$ sparse. The SCA is carried out using the two following approaches. The first one employs NMF algorithms, where mixing matrix $\mathbf{A}$ and source matrix $\mathbf{S}$ are estimated simultaneously. This is achieved usually through alternating least square methodology [22-25]. The second one referred in [16, 17, 32-34] breaks down BSS problem into two separate problems: estimation of the mixing matrix $\mathbf{A}$ and the number of materials $M$ using geometric concept known as data clustering [16-18, 32-34] and estimation of the materials or source matrix $\mathbf{S}$ (based on estimated A) by solving resulting underdetermined system of linear equations. This last step is carried out as linear 
accepted for Journal of Chemometrics

programming, $[26,32,35,36], \ell_{1}$-regularized least square problem, $[37,38]$ or $\ell_{2}$ regularized linear problem, [39].

To solve underdetermined BSS problem majority of clustering algorithms require that signal $\{\mathbf{s}(t)\}_{t=1}^{T}$ is $(N-1)$-sparse with $M-N+1$ zero components. By setting the number of mixtures to be $N=3$ (this corresponds with an RGB image) this implies $k=2$. It means that only two materials in the RGB image are allowed to occupy each pixel footprint. However, in blind multi-spectral image decomposition problem considered in this paper we shall even assume that materials present in the image are in average $k=1$ sparse. This implies that at each pixel footprint in average only one material is present. This assumption allows reduction of the computational complexity of to be described data clustering algorithm [17]. It is however correct assumption in considered problem of medical imaging of the skin tumors $[8,38]$. There, due to the small field of view the pixel footprint is very small (significantly less than $1 \mathrm{~mm}^{2}$ ). Thus, it is not reasonably to expect that more than one material will occupy such a small area (for examples see experimental RGB images of the skin tumor shown in Figures 5 and 7).

\subsection{Multilayer HALS NMF algorithm}

The HALS NMF algorithm minimizes global cost function to estimate mixing matrix A and set of local cost functions to estimate the unknown materials $\left\{\underline{\mathbf{s}}_{m}\right\}_{m=1}^{M}$. Global cost function employed for estimation of the mixing matrix is

$$
D(\mathbf{X} \| \mathbf{A S})=\frac{1}{2}\|\mathbf{X}-\mathbf{A} \mathbf{S}\|_{2}^{2}+\alpha_{\mathbf{S}} J_{\mathbf{S}}(\mathbf{S})+\alpha_{\mathbf{A}} J_{\mathbf{A}}(\mathbf{A})
$$


accepted for Journal of Chemometrics

where $J_{\mathbf{S}}(\mathbf{S})$ and $J_{\mathbf{A}}(\mathbf{A})$ represent sparseness constraint and $\alpha_{\mathbf{S}}$ and $\alpha_{\mathbf{A}}$ represent corresponding regularization constants. Because no constraints are imposed on $\mathbf{A}$ and because cost function (2) is minimized with respect to A only, both regularization constants in (2) are set to zero, i.e. $\alpha_{\mathbf{S}}=\alpha_{\mathbf{A}}=0$. To estimate matrix of materials $\mathbf{S}$ we employ minimization of the local cost functions $[23,25]$ :

$$
D^{(m)}\left(\mathbf{X}^{(m)} \| \mathbf{a}_{m} \underline{\mathbf{S}}_{n}\right)=\frac{1}{2}\left\|\mathbf{X}^{(m)}-\mathbf{a}_{m} \underline{\mathbf{S}}_{n}\right\|_{2}^{2}+\alpha_{\mathbf{s}}^{(m)} J_{\mathbf{S}}\left(\underline{\mathbf{s}}_{n}\right)+\alpha_{\mathbf{a}}^{(m)} J_{\mathbf{a}}\left(\mathbf{a}_{m}\right) \quad m=1, \ldots, M
$$

where $\mathbf{a}_{m}$ represent columns of $\mathbf{A}, \underline{\mathbf{s}}_{m}$ represent rows of $\mathbf{S}$ and $\mathbf{X}^{(m)}=\mathbf{X}-\sum_{j \neq m} \mathbf{a}_{j} \underline{\mathbf{s}}_{j}$. Because no constraints are imposed on $\mathbf{A}$ we set $\alpha_{\mathbf{a}}^{(m)}=0$. Since in considered blind multi-spectral image decomposition problem we have also assumed that materials in average do not overlap in spatial domain, sparseness constraint is imposed on them: $J_{\mathbf{S}}\left(\underline{\mathbf{s}}_{n}\right)=\sum_{t=1}^{T} S_{m t}$, where $\alpha_{s}^{(m)}$ is regularization constant. Local learning rule for rows of $\mathbf{S}$ and global learning rule for $\mathbf{A}$ is finally obtained as:

$$
\begin{aligned}
& \left\{\underline{\mathbf{s}}_{m} \leftarrow\left[\mathbf{a}_{m}^{\mathrm{T}} \mathbf{X}^{(m)}-\alpha_{\mathbf{s}}^{(m)} \mathbf{1}_{1 \times T}\right]_{+}\right\}_{m=1}^{M} \\
& \mathbf{A} \leftarrow\left[\mathbf{X} \mathbf{S}^{\mathrm{T}}\left(\mathbf{S S}^{\mathbf{T}}+\lambda \mathbf{I}_{M}\right)^{-1}\right]_{+} \\
& \left\{\mathbf{a}_{m} \leftarrow \mathbf{a}_{m} /\left\|\mathbf{a}_{m}\right\|_{2}\right\}_{m=1}^{M}
\end{aligned}
$$

where $\mathbf{I}_{M}$ is an $M \times M$ identity matrix, $\mathbf{1}_{1 \times T}$ is row vector with all entries equal to one and $[\xi]_{+}=\max \{\varepsilon, \xi\} \quad\left(\right.$ e.g., $\left.\varepsilon=10^{-16}\right)$. Regularization constant $\lambda$ changes as a function of the iteration index as: $\lambda_{k}=\lambda_{0} \exp (-k / \tau)$ (with $\lambda_{0}=20$ and $\tau=0.002$ in the experiments). 
accepted for Journal of Chemometrics

ALS concept implies that cost functions (3) and (4) are minimized in alternating fashion. Great improvement in the performance of the NMF algorithms is obtained when they are applied in the multilayer mode [26], whereas sequential decomposition of the nonnegative matrices is performed as follows. In the first layer, the basic approximation decomposition is performed $\mathbf{X} \cong \mathbf{A}^{(1)} \mathbf{S}^{(1)} \in \mathrm{R}_{0+}^{N \times T}$. In the second layer result from the first layer is used to build up new input data matrix for the second layer $\mathbf{X} \leftarrow \mathbf{S}^{(1)} \in \mathrm{R}_{0+}^{M \times T}$ yielding $\mathbf{X}^{(1)} \cong \mathbf{A}^{(2)} \mathbf{S}^{(2)} \in \mathrm{R}_{0+}^{M \times T}$. After $L$ layers the data decomposes as follows

$$
\mathbf{X} \cong \mathbf{A}^{(1)} \mathbf{A}^{(2)} \cdots \mathbf{A}^{(L)} \mathbf{S}^{(L)}
$$

Thus, learning rules (4) combined with multilayer mode of operation (5) is what constitutes multilayer HALS NMF algorithm.

\section{$3.2 \ell_{1}$-norm minimization algorithms}

Presuming the mixing matrix $\mathbf{A}$ is estimated through data clustering algorithm materials can be also estimated by means of linear programming

$$
\hat{\mathbf{s}}(t)=\underset{\mathbf{s}(t)}{\arg \min } \sum_{m=1}^{\hat{M}} S_{m}(t) \text { subject to } \hat{\mathbf{A}} \mathbf{s}(t)=\mathbf{x}(t) \quad \forall t=1, \ldots, T
$$

If the noise is present in blind decomposition problem more robust solution for $\{\mathbf{s}(t)\}_{t=1}^{T}$ is obtained by solving $\ell_{1}$-regularized least square problem, $[35,36]$ : 
accepted for Journal of Chemometrics

$$
\hat{\mathbf{s}}(t)=\underset{\mathbf{s}(t)}{\arg \min } \frac{1}{2}\|\hat{\mathbf{A}} \mathbf{s}(t)-\mathbf{x}(t)\|_{2}^{2}+\lambda\|\mathbf{s}(t)\|_{1} \quad \forall t=1, \ldots, T
$$

or $\ell_{2}$-regularized linear problem [37]:

$$
\hat{\mathbf{s}}(t)=\underset{\mathbf{s}(t)}{\arg \min }\|\mathbf{s}(t)\|_{1} \quad \text { subject to }\|\hat{\mathbf{A}} \mathbf{s}(t)-\mathbf{x}(t)\|_{2}^{2} \leq \varepsilon \quad \forall t=1, \ldots, T
$$

Note that all three formulations, (6) to (8), employed to solve resulting underdetermined system of linear equations are carried out on the pixel level. Hence, they are well suited for massively parallel implementation. Similarly, clustering algorithm to be described could be also parallelized by applying it on several disjoint pixel regions and then merging clustering results.

\subsection{Data clustering algorithm}

The importance of data clustering for HALS NMF algorithm and $\ell_{1}$-norm minimization algorithms is very different. In the former case it is only important for the estimation of the number of materials present in the image. In the later case it is also important for the estimation of the mixing matrix that is necessary for the solution of problems (6) to (8). Thus, final performance of blind image decomposition will be dependent much more on the accuracy of data clustering when $\ell_{1}$-norm minimization algorithms are employed than when HALS NMF algorithm is employed. This is an additional argument to advocate the use of HALS NMF algorithm in blind multispectral image decomposition.

Because solution of the BSS problem is generally characterized by scale indeterminacy we shall assume the unit norm constraint (in the sense of $\ell_{2}$ norm) on 
accepted for Journal of Chemometrics

the columns of the mixing matrix A, i.e., $\left\{\left\|\mathbf{a}_{m}\right\|_{2}=1\right\}_{m=1}^{M}$. Since we have assumed the number of mixtures to be $N=3$ the normalized mixing vectors $\left\{\mathbf{a}_{m}\right\}_{m=1}^{M}$ lie in a 3D space on the unit semi-sphere. They are parameterized as:

$$
\mathbf{a}_{m}=\left[\cos \left(\varphi_{m}\right) \sin \left(\theta_{m}\right) \sin \left(\varphi_{m}\right) \sin \left(\theta_{m}\right) \cos \left(\theta_{m}\right)\right]^{\mathrm{T}},
$$

where $\varphi_{m}$ and $\theta_{m}$ represent azimuth and elevation angles respectively. Due to the nature of the problem coefficients of the mixing vectors are nonnegative. Consequently the mixing angles $\varphi_{m}$ and $\theta_{m}$ are confined in the interval $[0, \pi / 2]$.

By assuming 1-dimensional concentration subspaces the clustering algorithm is outlined by the following steps:

1) We remove all data points close to the origin for which applies: $\left\{\|\mathbf{x}(t)\|_{2} \leq \varepsilon\right\}_{t=1}^{T}$, where $\varepsilon$ represents some predefined threshold. This corresponds to the case when all materials are close to zero.

2) Normalize to unit $\ell_{2}$ norm remaining data points $\mathbf{x}(t)$, i.e., $\left\{\mathbf{x}(t) \leftarrow \mathbf{x}(t) /\|\mathbf{x}(t)\|_{2}\right\}_{t=1}^{\bar{T}}$, where $\bar{T} \leq T$ denotes number of data points that remained after elimination process.

3) Calculate function $f(\mathbf{a})$, where $\mathbf{a}$ is defined with (9):

$$
f(\mathbf{a})=\sum_{t=1}^{\bar{T}} \exp \left(-\frac{d^{2}(\mathbf{x}(t), \mathbf{a})}{2 \sigma^{2}}\right)
$$

where $d(\mathbf{x}(t), \mathbf{a})=\sqrt{1-(\mathbf{x}(t) \cdot \mathbf{a})^{2}}$ and $(\mathbf{x}(t) \cdot \mathbf{a})$ denotes inner product. Parameter $\sigma$ in (10) is called dispersion. If set to sufficiently small value, in our experiments this 
accepted for Journal of Chemometrics

turned out to be $\sigma \approx 0.05$, the value of the function $f(\mathbf{a})$ will approximately equal the number of data points close to a. Thus, by varying mixing angles $0 \leq \varphi, \theta \leq \pi / 2$ we effectively cluster data.

4) Number of peaks of the function $f(\mathbf{a})$ corresponds with the estimate of the number of materials $\hat{M}$. Locations of the peaks correspond with the estimates of the mixing angles $\left\{\left(\hat{\varphi}_{m}, \hat{\theta}_{m}\right)\right\}_{m=1}^{\hat{M}}$, i.e., mixing vectors $\left\{\hat{\mathbf{a}}_{m}\right\}_{m=1}^{\hat{M}}$, where $\hat{\mathbf{a}}_{m}$ is given with (9). The hat sign introduced here is used to denote estimate of the related quantity. Hence, at the end of data clustering phase estimates of the number of materials $\hat{M}$ and mixing matrix $\mathbf{A}$ are obtained.

From above exposition it is evident that value of dispersion constant $\sigma$ in (10) will influence the number of clusters. Therefore, in real world situations the number of clusters will most likely be either over- or underestimated. Practical experience with certain type of data sets will help in proper selection of the parameter $\sigma$. Generally, if the number of clusters is underestimated, spectrally similar materials will be extracted (segmented) together as one material. If the number of clusters is overestimated some materials will be extracted several times. This later case is less dangerous than the former one, because in the former case some materials are lost. Existence of multiple materials is checked easily by calculating how much they are mutually correlated. Thus, one way to circumvent difficulty associated with under- or overestimation of the number of clusters is to run the algorithm twice with two different values of dispersion constant $\sigma$ and check whether new mutually uncorrelated spatial maps will be extracted or some of the extracted maps will be repeated.

\section{EXPERIMENTAL RESULTS}


accepted for Journal of Chemometrics

Two examples related to blind decomposition of experimental RGB images are used for comparative performance analysis between: multilayer HALS NMF algorithm (4) and (5) $[24,27]$, linear programming method used to solve problem (6) and interiorpoint method [37] used to solve problem (7). The principal reason for using RGB images and not hyperspectral images for performance evaluation is that underdetermined BSS problem is very likely to happen in this low-dimensional multispectral case. Linear programming algorithm is implemented by MATLAB command linprog, while MATLAB code for interior-point method has been provided at [41]. To cope with the eventual presence of noise in the experimental RGB image regularization constant in (7) was set to $\lambda=10$. This is due to the fact that optimal value of the regularization constant is proportional to the noise variance [40, 41]. Both examples were implemented in MATLAB environment on $2.4 \mathrm{GHz}$ Intel Core 2 Quad Processor Q6600 based desktop computer with 4GB RAM.

\subsection{Experiment 1: RGB image consisting of four materials}

Blind decomposition of the experimental RGB image shown in Figure 1 that consists of four materials is underdetermined BSS problem. Because materials in the experimental RGB image do not overlap in spatial domain we can evaluate performance of the employed blind image decomposition methods via the correlation matrix defined as $\mathbf{G}=\mathbf{S S}^{\mathrm{T}}$. For the perfect separation the correlation matrix should be diagonal. Hence, separation performance can be visualized as deviation from diagonal matrix (see Figure 4). To quantify decomposition quality numerically we compute the correlation index in $\mathrm{dB}$ scale as

$$
C R=-10 \log _{10} \sum_{\substack{i, j=1 \\ j \neq i}}^{M} g_{i j}^{2}
$$


accepted for Journal of Chemometrics

where before calculating correlation matrix $\mathbf{G}$ rows of $\mathbf{S}$ are normalized to unit $\ell_{2}$ norm. To make the extracted spatial maps of the materials visually comparable we have rescaled each extracted material to the interval $[0,1]$ and shown it in pseudo color scale. Dark blue color represents 0, i.e. the absence of the material and dark red color represents 1, i.e. the presence of the material. Hence, quality of the image decomposition is visible immediately. Figure 2 shows clustering function, eq.(10), in the domain of mixing angles with the dispersion constant $\sigma=0.05$. Four clusters confirm existence of four materials present in the RGB image.

Multilayer HALS NMF algorithm [23, 26], with sparseness regularization constraint $\alpha_{s}^{(m)}=0.5,25$ layers and 500 iterations per layer has been applied to perform blind image decomposition task. Figure 3 shows decomposition results obtained by multilayer HALS NMF algorithm, interior point method and linear programming method. Corresponding correlation matrices are shown in Figure 4. Numerical values of the correlation index in $\mathrm{dB}$ and computation times in seconds are given in Table 1. Multilayer HALS NMF algorithm outperformed the other two SCA approaches in term of the separation quality keeping at the same time the same level of computational complexity. Size of the RGB image shown in Figure 1 was $T=384 \times 512=196608$ pixels. Computation times are estimated only for multilayer HALS NMF algorithm (4) and (5), interior-point method employed to solve (7) and linear program employed to solve (6). Computation time of data clustering algorithm [17] was not taken into account because all three SCA algorithms under consideration used results of data clustering algorithm for their inputs. Thus, data clustering method contributed to the computational complexity of all three methods equally. 
accepted for Journal of Chemometrics

\subsection{Experiment 2: RGB fluorescent images of the skin tumor}

We now execute comparative performance evaluation of the presented methods on the RGB fluorescent image of the skin tumor (basal cell carcinoma) [38]. For this purpose an image of a skin tumor is recorded after the tumor was treated for $4 \mathrm{~h}$ with $\delta$-5aminolaevulinic acid (ALA). ALA is a photo-synthesiser that, through the process of biosynthesis, causes formation of the fluorophore protoporphyrin IX (PpIX) [42]. The tumor was illuminated with $405 \mathrm{~nm}$ light, which induces fluorescence of the PpIX. The fluorescent image was recorded by camera with an attached filter used to filter out the reflected $405 \mathrm{~nm}$ light. In order to evaluate robustness of the image decomposition methods against variation of the intensity of fluoresce, fluorescent RGB image of the tumor has been acquired after illumination with high-intensity light (shown in Figure 5) as well as after illumination with weak-intensity light (shown in Figure 7). Size of the images shown in Figures 5 and 7 was $T=856 \times 1144=979264$ pixels. The high-intensity fluorescent image shown in Figure 5 was used to extract spatial binary maps of the tumor and surrounding healthy tissue. They served as a ground truth for calculation of the receiver-operating-characteristic (ROC) curves necessary to quantify performance of the SCA algorithms. Like in Figure 1, fluorescent RGB images shown in Figure 5 and 7 also contain four materials. They are: basal cell carcinoma, surrounding healthy tissue, ruler that was inserted into the scene to give perspective about the spatial dimensions of the tumor and border region between tumor and surrounding tissue that is spectrally distinct from both tumor and healthy tissue. Figure 6 shows spatial distributions of these materials extracted by multilayer HALS NMF algorithm [23, 26] with sparseness regularization constraint $\alpha_{s}^{(m)}=0.5,25$ layers and 500 iterations per layer. Like in example 4.1, extracted spatial maps are rescaled to the interval $[0,1]$ and shown in pseudo color scale. Image in the upper right corner of the Figure 6 represents spatial map of the basal cell carcinoma. All four extracted spatial maps have meaningful interpretation and are consistent with our knowledge of the structure of the RGB image shown in Figure 5. Extraction of the tumor spatial map with the stable boundary from the low-intensity fluorescent image (shown in Figure 7) was a challenge 
accepted for Journal of Chemometrics

for blind decomposition methods. Figures $8 \mathrm{a}$ to $8 \mathrm{c}$ respectively show spatial maps of the tumor extracted from RGB image shown in Figure 7 by means of: multilayer HALS NMF algorithm (4) and (5) with sparseness regularization constraint $\alpha_{s}^{(m)}=0.1,5$ layers and 1000 iterations per layer; interior point method (7) and linear programming method (6). Multilayer HALS NMF algorithm yielded significantly larger separation margin between the tumor material and the rest of the image than other two methods. This is confirmed in Figure 9 which shows ROC curves for the three methods. For the same probability of false alarm the HALS NMF algorithm yields greater probability of detection.

\section{CONCLUSION}

Multilayer hierarchical alternating least square nonnegative matrix factorization algorithm is proposed for blind decomposition of low-dimensional multi-spectral image. Performance of the algorithm is invariant with respect to statistical (in)dependence among materials present in the image which is not the case with the majority of BSS methods. Unlike many other BSS methods, proposed algorithm is capable to estimate the unknown number of materials present in the image as well as to extract spatial distributions of the materials when the number of materials is greater than the number of spectral bands. Outlined capabilities have been experimentally demonstrated on RGB images with the known ground truth. It has been demonstrated quantitatively through comparative performance analysis, that multilayer HALS NMF algorithm outperforms other state-of-the-art methods in solving underdetermined BSS problems: interior point method and linear programming method. In addition to tumor demarcation problem that is demonstrated in the paper, other areas that can also benefit 
accepted for Journal of Chemometrics

from proposed method are cell and chemical imaging with application in chemistry, biology and medicine.

\section{ACKNOWLEDGMENTS}

This work was supported through grant 098-0982903-2558 funded by the Ministry of Science, Education and Sports, Republic of Croatia. The authors wish to thank anonymous reviewers for very constructive comments that contributed to the quality of the paper significantly. The authors also thank Ivanka Jerić and David Nagel for proofreading the final version of the manuscript.

\section{REFERENCES}

1. Adams J.B., Smith M.O. Spectral mixture modeling: A new analysis of rock and soil types at the Viking Lander 1 suite. J. Geophys. Res. 1986; 91: 8098-8112.

2. Settle J.J., Drake N.A. Linear mixing and estimation of ground cover proportions. Int. J. Remote Sens. 1993; 14: 1159-1177.

3. Chang C.-I., Chiang S.-S., Smith J.A., Ginsberg I.W. Linear spectral random mixture analysis for hyperspectral imagery. IEEE Trans. Geosci. Remote Sensing 2002; 40: $375-392$.

4. Du Q., Kopriva I., Szu H. Independent-component analysis for hyperspectral remote sensing imagery classification. Opt. Eng. 2006; 45: 017008:1-13.

5. Du Q. Kopriva I. Automated Target Detection and Discrimination Using Constrained Kurtosis Maximization. IEEE Geosci. Remote Sens. Lett. 2008; 5: 38-42.

6. Stein D.W.J., Beaven S.G., Hoff, L.E., Winter, E.M., Schaum, A.P., Stocker, A.D. Anomaly detection from hyperspectral imagery. IEEE Signal Process. Mag. 2002; 19: 58-69. 
accepted for Journal of Chemometrics

7. Zavattini G., Vecchi S., Mitchell G., Weisser U., Leahy R.M., Pichler B.J., Smith D.J., Cherry S.J. A hyperspectral fluorescence system for 3D in vivo optical imaging. Phys. Med. Biol. 2006; 51: 2029-2043.

8. Kopriva I., Peršin A., Zorc H., Lipozenčić J., Pašić A., Kostović K., Lončarić M. Visualization of basal cell carcinoma by fluorescence diagnosis and independent component analysis. Photodiagnosis and Photodynamic Therapy 2007; 4: 190-196.

9. Hyvärinen A., Karhunen J., Oja E. Independent Component Analysis, John Wiley \& Sons, Inc.: New York, US, 2001.

10. Cichocki A., Amari S. Adaptive Blind Signal and Image Processing, John Wiley: New York, 2002.

11. Jutten C., Herault, J. Blind separation of sources, part I: An adaptive algorithm based on neuromimetic architecture. Sig. Proc. 1991; 24: 1-10.

12. Comon P. Independent component analysis - A new concept?. Sig. Proc. 1994; 36: $287-314$

13. Cardoso J.F., Soulomniac A. Blind beamforming for non-Gaussian signals. Proc. IEE F 1993; 140: 362-370.

14. Nascimento J.M.P., Bioucas Dias, J.M. Does Independent Component Analysis Play a Role in Unmixing Hyperspectral Data? IEEE Trans. Geosci. Remote Sensing 2005; 43: 175-187.

15. Li Y., Cichocki, A., Amari, S. Analysis of Sparse Representation and Blind Source Separation. Neural Comput. 2004; 16: 1193-1234.

16. Li Y., Amari S., Cichocki A., Ho D.W.C., Xie S. Underdetermined Blind Source Separation Based on Sparse Representation. IEEE Trans. Signal Process. 2006; 54: 423-437. 
accepted for Journal of Chemometrics

17. Naini F.M., Mohimani G.H., Babaie-Zadeh M., Jutten C. Estimating the mixing matrix in Sparse Component Analysis (SCA) based on partial $k$-dimensional subspace clustering. Neurocomputing 2008; 71: 2330-2343.

18. Nascimento J.M.P., Bioucas Dias J.M. Vertex Component Analysis: A Fast Algorithm to Unmix Hyperspectral Data. IEEE Trans. Geosci. Remote Sensing 2005; 43: 898-910.

19. Chan T.H., Ma W.K., Chi Ch. Y., Wang Y. A Convex Analysis Framework for Blind Separation of Non-Negative Sources. IEEE Trans. Signal Process. 2008; 56: $5120-5134$.

20. Lee D.D., Seung H.S. Learning the parts of objects by non-negative matrix factorization. Nature 1999; 401: 788-791.

21. Cichocki A., Zdunek R., Amari S. Nonnegative Matrix and Tensor Factorization. IEEE Signal Process. Mag. 2008; 25: 142-145.

22. Zdunek R., Cichocki A. Nonnegative matrix factorization with constrained secondorder optimization. Sig. Proc. 2008; 87: 1904-1916.

23. Cichocki A., Zdunek R., Amari S.I. Hierarchical ALS Algorithms for Nonnegative Matrix Factorization and 3D Tensor Factorization. LNCS 2007; 4666: 169-176.

24. Cichocki A., Phan A-H., Zdunek R., Zhang, L-Q. Flexible component analysis for sparse, smooth, nonnagative coding or representation. LNCS 2008; 4984: 811-820.

25. Cichocki A., Zdunek, R., Phan A.H., Amari. S. Nonnegative Matrix and Tensor Factorization, John Wiley \& Sons, Ltd., New York, US, 2009.

26. Cichocki A., Zdunek R. Multilayer nonnegative matrix factorization. Electron. Lett. 2006; 42: 947-948.

27. Levina E., Wagaman A.S., Callender A.F., Mandair G.S., Morris M.D. Estimating the number of pure chemical components in a mixture by maximum likelihood. $J$. Chemometr. 2007; 21: 24-34. 
accepted for Journal of Chemometrics

28. Malinowski E.R. Determination of the number of factors and experimental error in a data matrix. Anal. Chem. 1977; 49: 612-617.

29. Chang C.I., Du Q. Estimation of number of spectrally distinct signal sources in hyperspectral imagery. IEEE Trans. Geosci. Remote Sensing 2004; 42: 608-619.

30. Luengo D., Santamaria I., Vielva L. A general solution to blind inverse problems for sparse input signal. Neurocomputing 2005; 69: 198-215.

31. Georgiev P., Theis F., Cichocki A. Sparse Component Analysis and Blind Source Separation of Underdetermined Mixtures. IEEE Trans. Neural Netw. 2005; 16: $992-$ 996.

32. Bofill, P., Zibulevsky, M. Underdetermined blind source separation using sparse representations. Sig. Proc. 2001; 81: 2353-2362.

33. Takigawa I., Kudo M, Toyama J. Performance Analysis of Minimum $l_{1}$-Norm Solutions for Underdetermined Source Separation. IEEE Trans. Signal Process. 2004; 52: $582-591$.

34. Donoho D.L., Elad M. Optimally sparse representation in general (non-orthogonal) dictionaries via $l_{1}$ minimization. Proc. Nat. Acad. Sci. USA 2003; 100: 2197-2202.

35. Kim S.J., Koh K., Lustig M., Boyd S., Gorinevsky S. An Interior-Point Method for Large-Scale $\ell_{1}$-Regularized Least Squares. IEEE J. Selected Topics Sig. Proc. 2007; 1: 606-617.

36. Tropp J.A., Gilbert A.C. Signal Recovery from Random Measurements via Orthogonal Matching Pursuit. IEEE Trans. Inf. Theory 2007; 53: 4655-4666.

37. Figuiredo M.A.T., Nowak R.D., Wright S.J. Gradient Projection for Sparse Reconstruction: Application to Compressed Sensing and Other Inverse Problems. IEEE J. Selected Topics Sig. Proc. 1 2007; 586-597. 
accepted for Journal of Chemometrics

38. Kopriva I., Peršin A. Unsupervised decomposition of low-intensity lowdimensional multi-spectral fluorescent images for tumour demarcation, Med. Image Anal. 13 2009; 507-518.

39. http://www.stanford.edu/ boyd/11_ls/.

40. Chen S., Donoho D., Saounders M. Atomic decomposition by basis pursuit. SIAM J. Sci. Comput. 1998; 20: 33-61.

41. Van Den Berg E., Friedlander M.P. Probing the Pareto Frontier for Basis Pursuit Solutions. SIAM J. Sci. Comput. 2008; 31: 890-912.

42. Koenig F., Knittel J., Stepp H. Diagnostic cancer in vivo. Science 2001; 292: 14011403. 
accepted for Journal of Chemometrics

\section{Figure Captions}

Figure 1. Experimental RGB image consisted of four different materials.

Figure 2. Clustering function (10) in the domain of mixing angles for the RGB image shown in Figure 1. Dispersion constant was $\sigma=0.05$. Four clusters confirm existence of the four materials in the RGB image.

Figure 3. Spatial maps of the materials extracted from the RGB image shown in Figure 1 by means of: a) multilayer HALS NMF algorithm [23-26] with 25 layers, 500 iterations per layer and sparseness constraints $\alpha_{s}^{(m)}=0.5$; b) interior point method [35, 39]; c) linear programming. Extracted materials were rescaled to the interval $[0,1]$ and shown it in pseudo color scale, wherein dark blue color represents 0 , i.e. the absence of the material, and dark red color represents 1, i.e. the presence of the material. (For interpretation of the references to color in this figure legend, the reader is referred to the web version of this article.)

Figure 4. Correlation maps of the extracted sources. From left to right: multilayer HALS NMF algorithm [23-26] with 25 layers, 500 iterations per layer and sparseness constraints $\alpha_{s}^{(m)}=0.5$, interior point method [35,39], and linear programming.

Figure 5. RGB fluorescent image of the skin tumor acquired after illumination with high-intensity light.

Figure 6. Spatial maps of the materials extract from fluorescent RGB image shown in Figure 5 by means of multilayer HALS NMF algorithm [23-26] with 25 layers, 500 iterations per layer and sparseness constraints $\alpha_{s}^{(m)}=0.5$.

Figure 7. RGB fluorescent image of the skin tumor acquired after illumination with the light of weak intensity.

Figure 8. Spatial maps of the tumor extracted from fluorescent RGB image shown in Figure 7. a) multilayer HALS NMF algorithm [23-26] with 5 layers, 1000 iterations 
accepted for Journal of Chemometrics

per layer and sparseness constraints $\alpha_{s}^{(m)}=0.1$; b) interior point method [35,39]; c) linear programming. Extracted materials were rescaled to the interval $[0,1]$ and shown it in pseudo color scale, wherein dark blue color represents 0 , i.e. the absence of the material, and dark red color represents 1, i.e. the presence of the material. (For interpretation of the references to color in this figure legend, the reader is referred to the web version of this article).

Figure 9. ROC curves calculated for spatial maps of the tumor shown in Figure 8 according to legend: blue circles - multilayer HALS NMF algorithm; green squares linear programming method; red diamonds - interior point method. (For interpretation of the references to color in this figure legend, the reader is referred to the web version of this article). 
accepted for Journal of Chemometrics

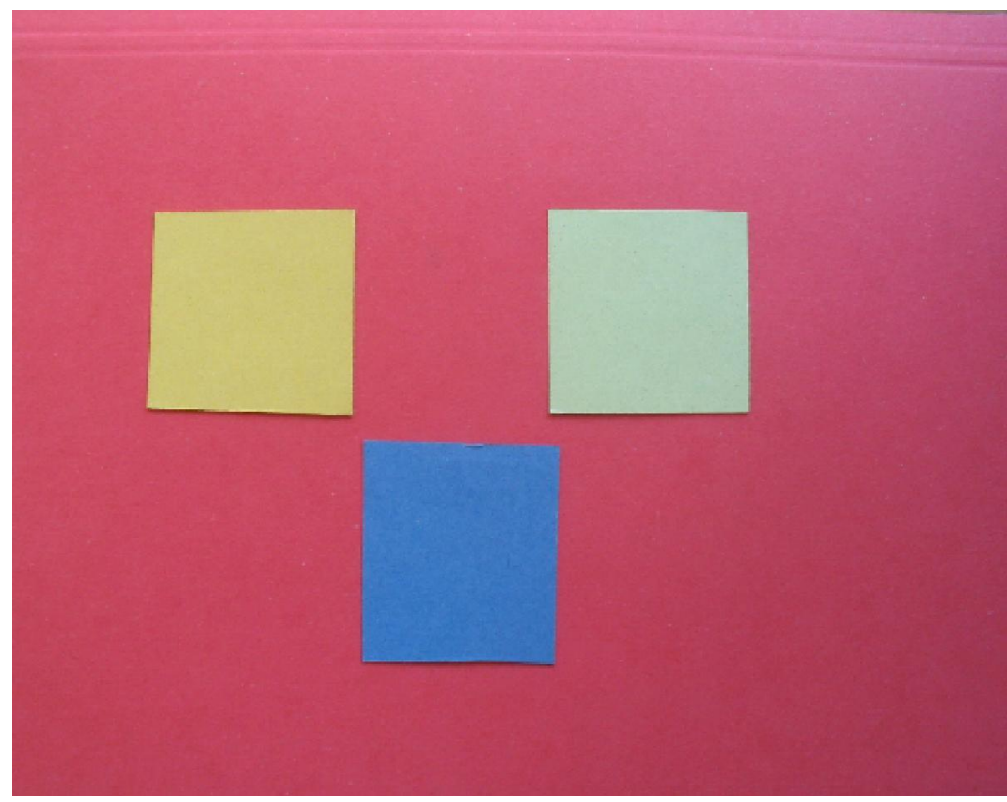

Fig. 1, Kopriva \& Cichocki

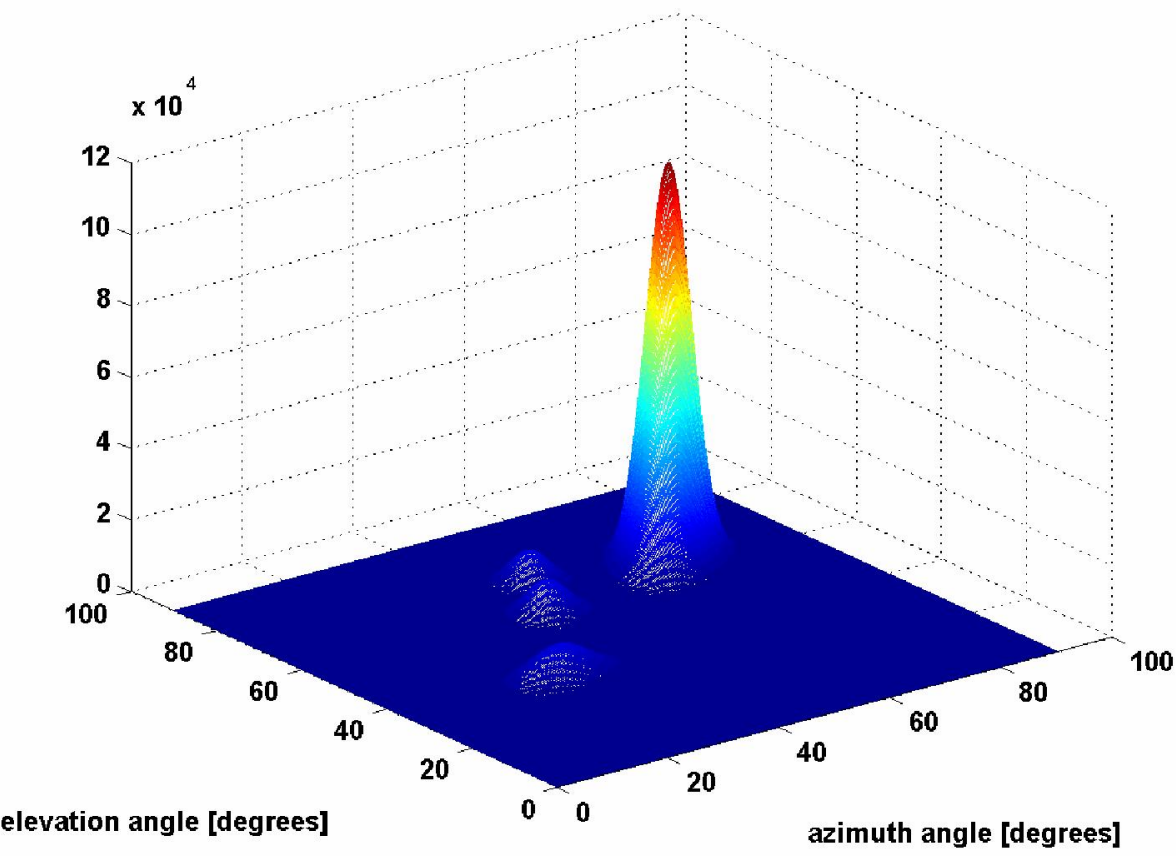

Fig. 2, Kopriva \& Cichocki 
accepted for Journal of Chemometrics

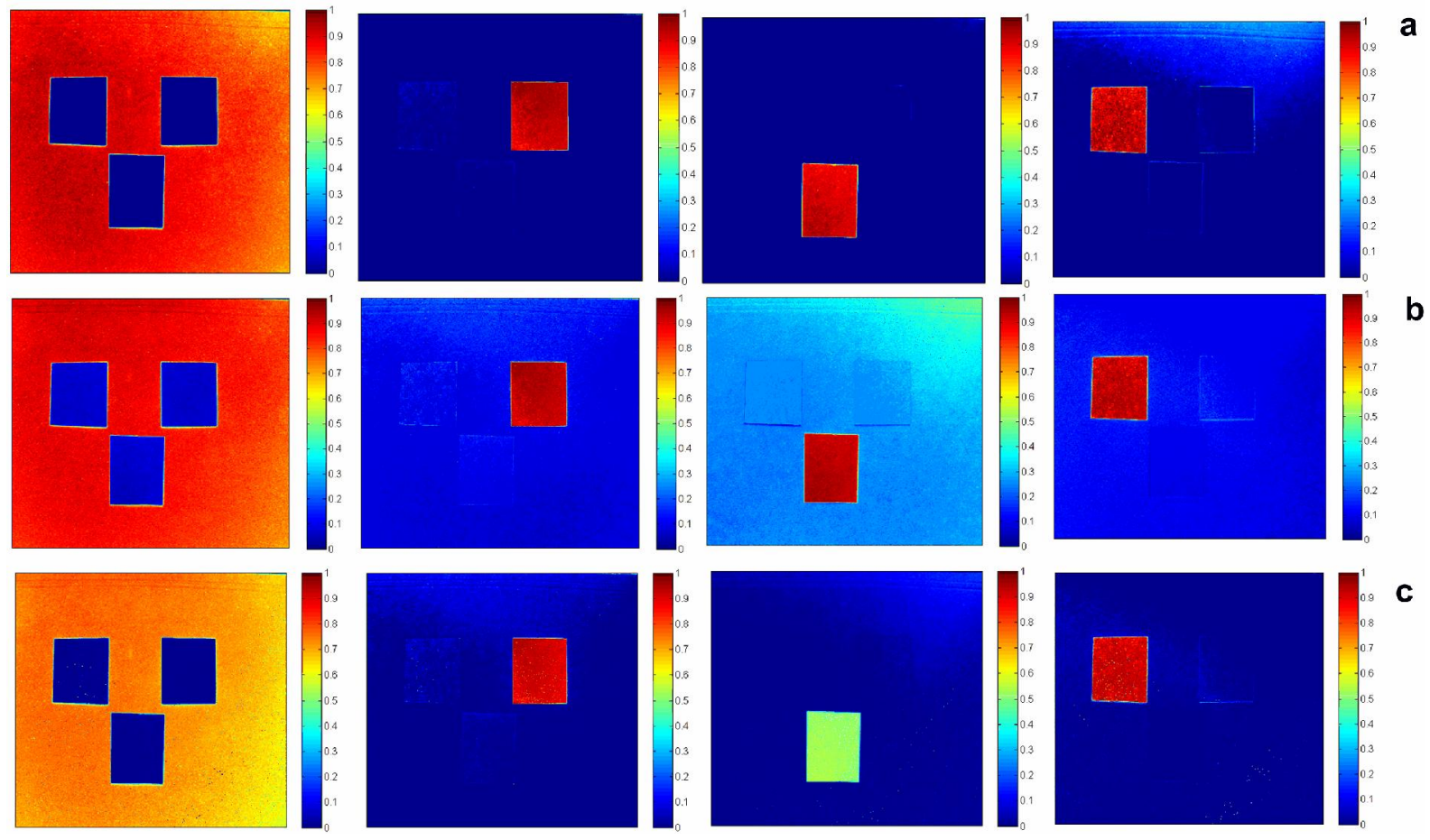

Fig. 3, Kopriva \& Cichocki
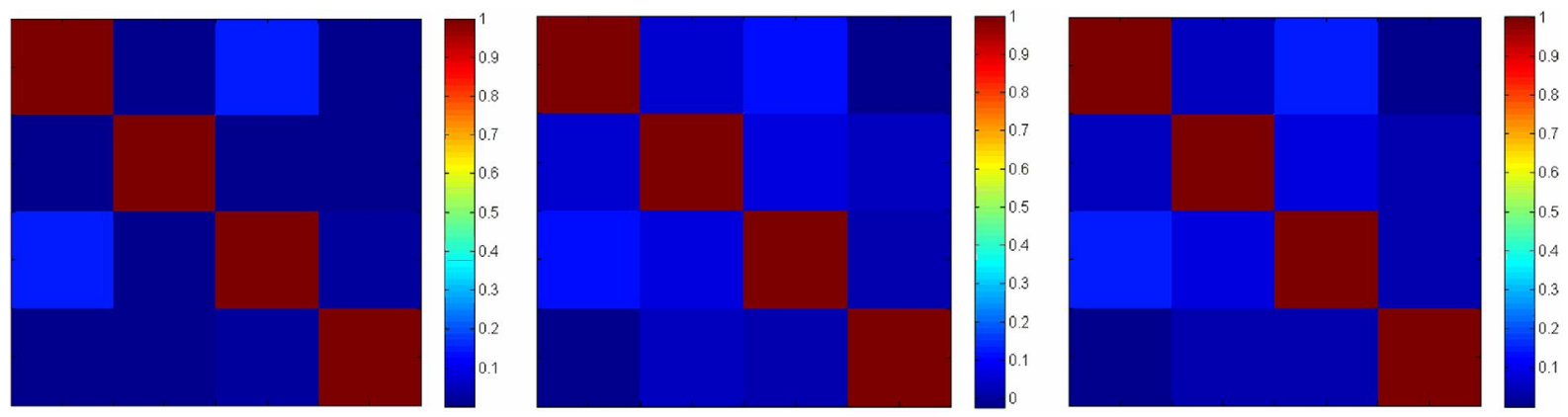

Fig. 4, Kopriva \& Cichocki 
accepted for Journal of Chemometrics

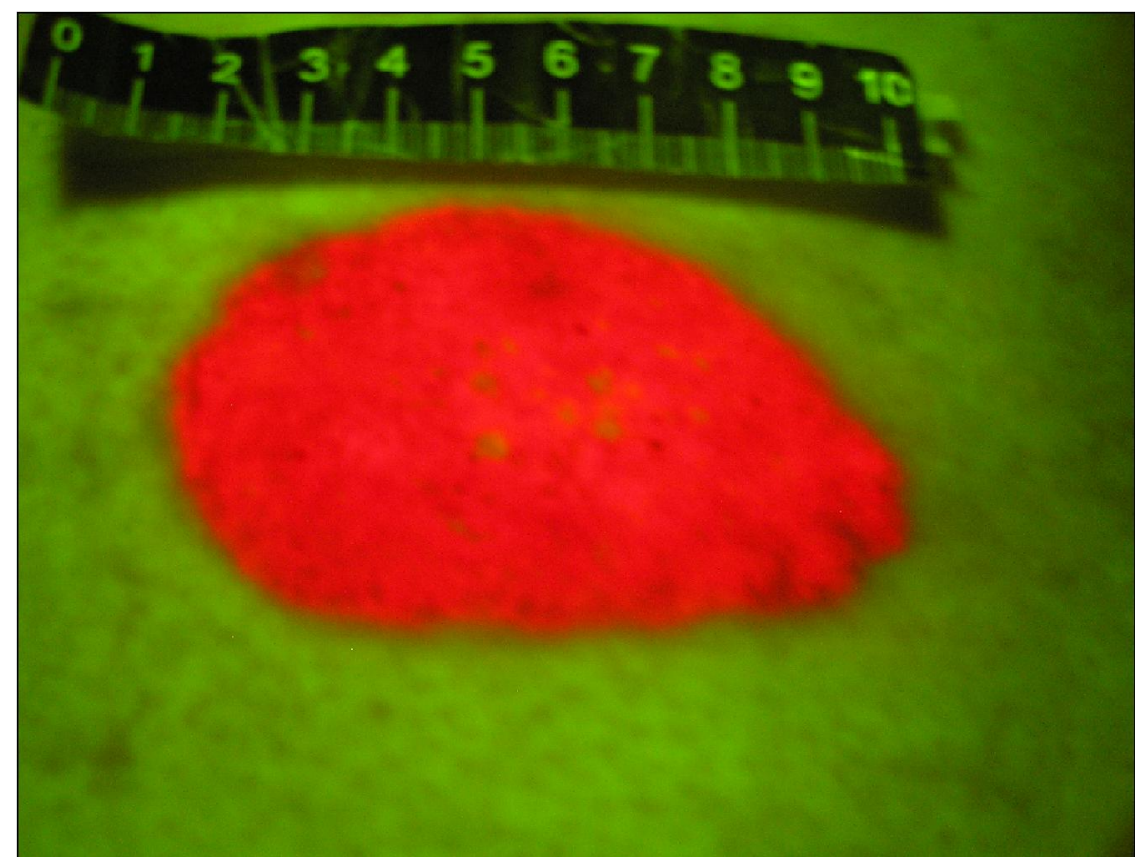

Fig. 5, Kopriva \& Cichocki
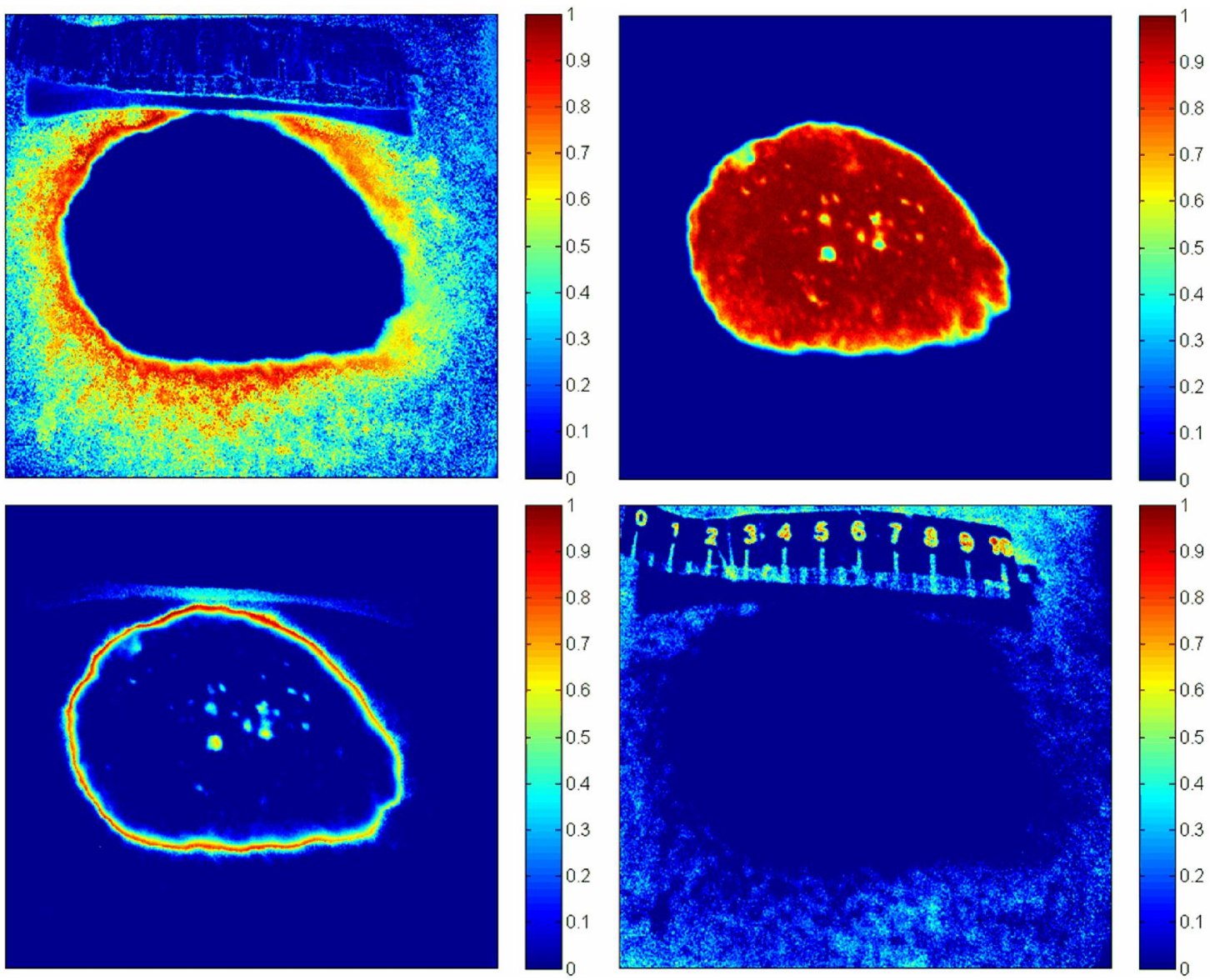

Fig. 6, Kopriva \& Cichocki 
accepted for Journal of Chemometrics

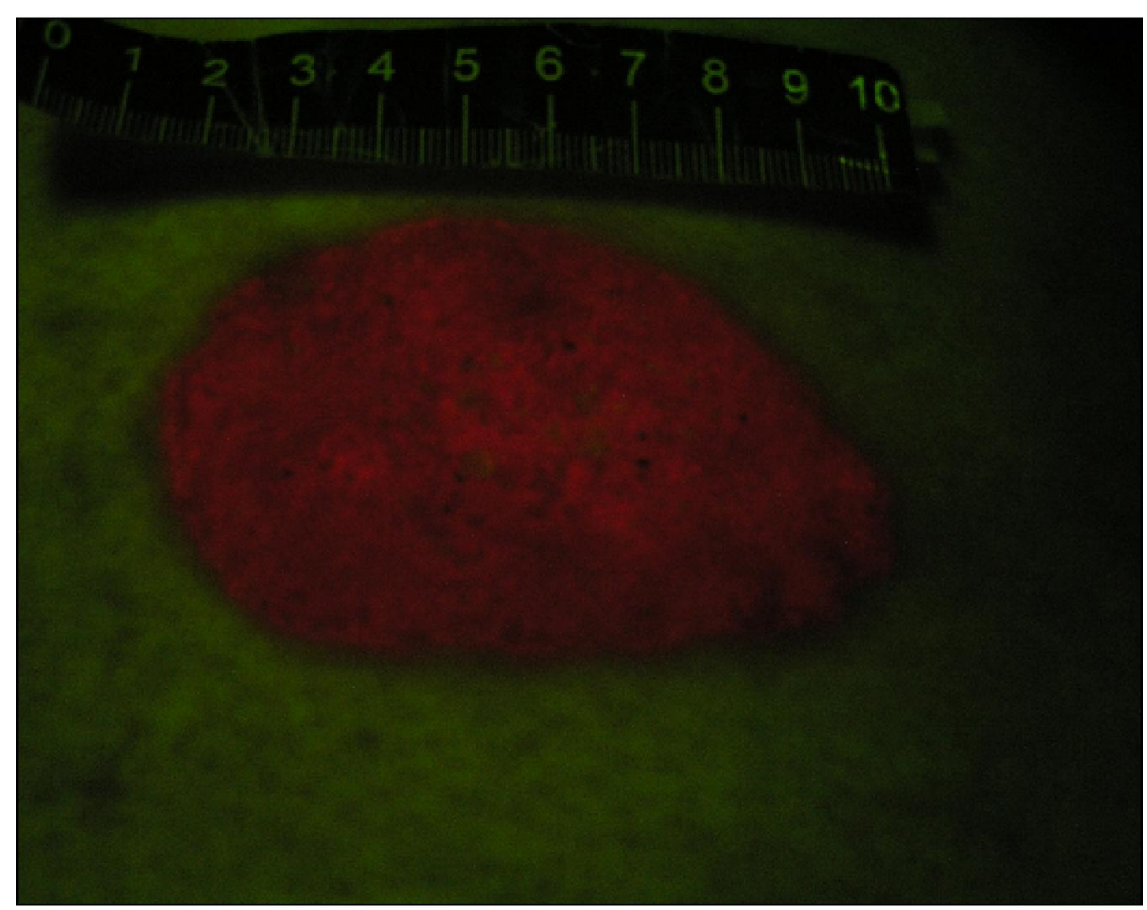

Fig. 7, Kopriva \& Cichocki
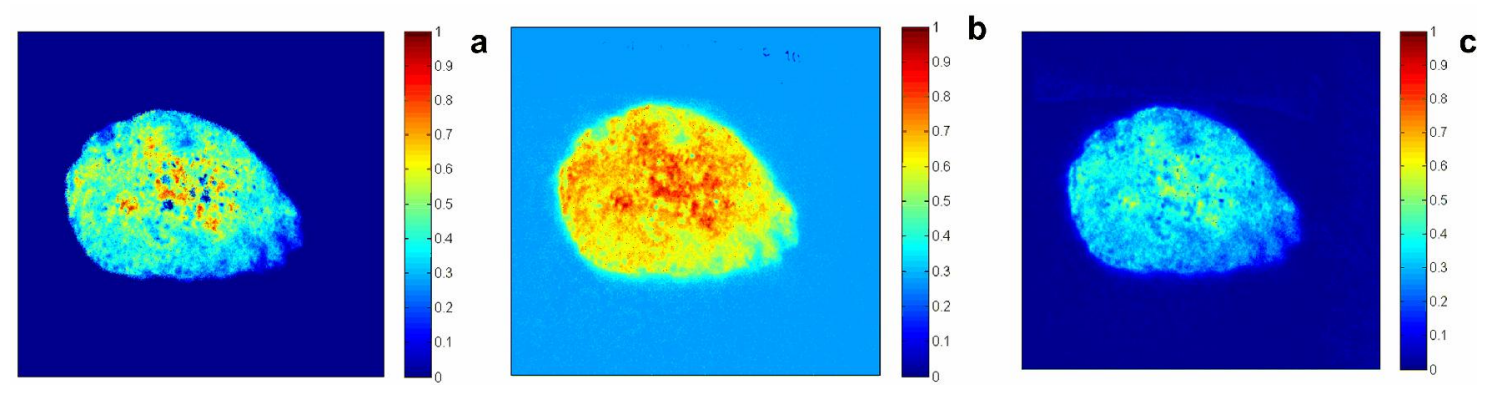

Fig. 8, Kopriva \& Cichocki 
accepted for Journal of Chemometrics

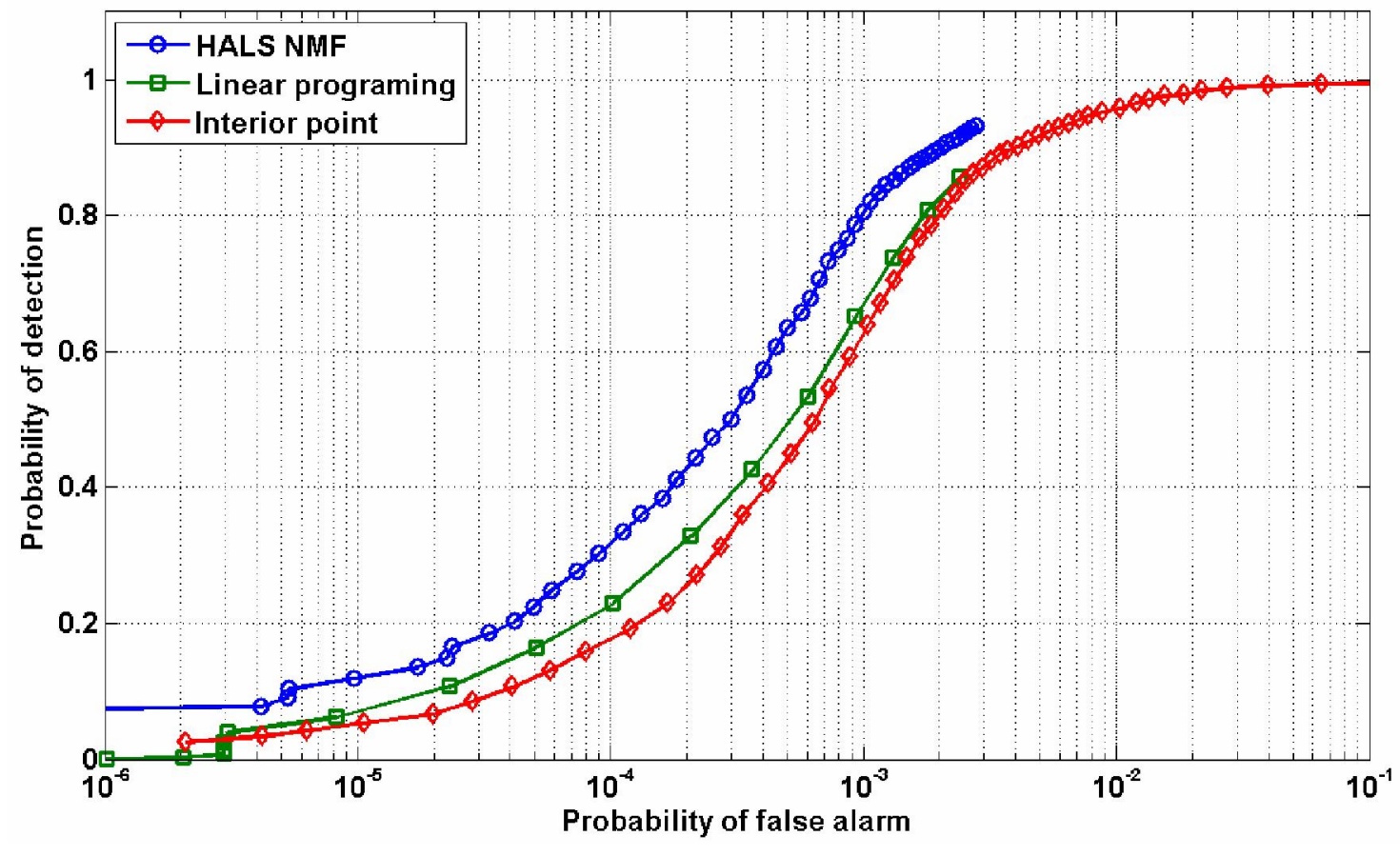

Fig. 9, Kopriva \& Cichocki 
accepted for Journal of Chemometrics

\section{Table Captions}

Table 1. Rows: CR performance, eq. (11), and computational time for: multilayer HALS NMF algorithm, [30-32]; $\ell_{1}$-regularized least square problem, [44,52]; linear programming. Columns: Type of algorithm employed to solve blind RGB image decomposition problem.

\begin{tabular}{cccc}
\hline & $\begin{array}{c}\text { Multilayer HALS NMF } \\
\text { (6) and (7) }\end{array}$ & $\begin{array}{c}\text { Interior-point method } \\
\text { (9) }\end{array}$ & Linear program (8) \\
\hline CR [dB] & 13.67 & 9.97 & 7.77 \\
\hline CPU time [s] & 3097 & 7751 & 3265 \\
\hline
\end{tabular}

Table 1, Kopriva \& Cichocki 
accepted for Journal of Chemometrics

\title{
Table of Content Abstract
}

Title: Blind decomposition of low-dimensional multi-spectral image by sparse component analysis

\section{Ivica Kopriva ${ }^{1}$, and Andrzej Cichocki ${ }^{2,3}$}

${ }^{1}$ Division of Laser and Atomic Research and Development, Rudjer Bosković Institute Bijenička cesta 54, HR-10000, Zagreb, Croatia phone: +385-1-4571-286, fax:+385-1-4680-104 e-mail: ikopriva@irb.hr

${ }^{2}$ Laboratory for Advanced Brain Signal Processing

Brain Science Institute, RIKEN 2-1Hirosawa, Wako-shi, Saitama, 351-0198, Japan

${ }^{3}$ Warsaw University of Technology and Systems Research Institute, PAN, Poland. e-mail: cia@brain.riken.jp

\begin{abstract}
Multilayer hierarchical alternating least square nonnegative matrix factorization approach has been applied to blind decomposition of low-dimensional multi-spectral image. Performance of the algorithm is invariant with respect to statistical (in)dependence between materials present in the image that is an assumption upon which many existing blind source separation methods depend. The proposed method performs blind decomposition exploiting spectral diversity and spatial sparsity between the materials present in the image. Unlike many existing blind source separation methods the method is capable to estimate the unknown number of materials present in the image. This number can be less than, equal to or greater than the number of spectral
\end{abstract}


accepted for Journal of Chemometrics

bands. Performance of the method is evaluated on underdetermined blind source separation problems associated with blind decompositions of experimental red-greenblue images composed of four materials. The proposed algorithm showed best performance in comparison with methods based on $\ell_{1}$-norm minimization: linear programming and interior-point methods. In addition to tumor demarcation problem demonstrated in the paper, other areas that can also benefit from proposed method are cell and chemical imaging. 